\title{
Pancreatic triacylglycerol distribution in type 2 diabetes
}

\author{
Kieren G. Hollingsworth ${ }^{1} \cdot$ Ahmad Al-Mrabeh $^{1} \cdot$ Sarah Steven $^{1} \cdot$ Roy Taylor $^{1}$
}

Received: 29 May 2015 / Accepted: 15 July 2015 /Published online: 2 August 2015

(C) Springer-Verlag Berlin Heidelberg 2015

Keywords Ectopic fat $\cdot$ Magnetic resonance imaging · Pancreas $\cdot$ Pancreatic steatosis $\cdot$ Type 2 diabetes

\section{Abbreviation \\ MR Magnetic resonance}

To the Editor: The paper by Begovatz and colleagues concluded that there was no fat in the parenchymal tissue of the pancreas, and hence local release of fatty acids could not influence endocrine function [1]. The authors describe intralobular fat within the pancreas as detected by spectroscopy but then concluded that there was no fat in the parenchyma of the pancreas. This conclusion relied upon magnetic resonance (MR) techniques. We draw attention to three serious errors in the MR methodology employed, and to the sound evidence for the existence of parenchymal fat.

First, the imaging method used to measure parenchymal fat yielded negative percentages for pancreas parenchymal fat in approximately half of all individuals, the range of individual parenchymal fat appearing to be from $-3 \%$ to $+4 \%$. Negative fat content of tissue is a concept of no biological validity. The

Roy Taylor

roy.taylor@ncl.ac.uk

1 Magnetic Resonance Centre, Institute of Cellular Medicine, Campus for Ageing \& Vitality, Newcastle University, Newcastle upon Tyne NE4 5PL, UK lower limit of detection is stated by the authors as $2 \%$, and from Fig. 21 in their paper it can be seen that this means the majority of their observations cannot yield what they themselves define as a meaningful result. There appears to be a problem of calibration at low fat fraction, possibly caused by the noise performance of fitting too many variables to a twopoint Dixon acquisition, rather than using three or more echoes. Although the authors claim that the technique used has been validated, their supporting reference 29 is an abstract that relates to the simpler matter of measuring liver fat content.

Second, it is suggested that there is no fat in the parenchyma of the pancreas but that fat within the pancreas in type 2 diabetes is contained in thick bands of adipose tissue. We have thoroughly assessed over 100 pancreases of people with diabetes and 32 with normal glucose tolerance using our published three-point Dixon method [2]. As illustrated by six examples in our Fig. 1, fat is distributed at a low level throughout each pancreas, and localised very high concentrations of fat are rare within the organ. One of the advantages of the three-point Dixon technique is that it is possible to study smaller organs with precise knowledge of boundaries [3]. We have previously reported that the pancreas is $30 \%$ smaller in volume and has a more serrated border in type 2 diabetes compared with age-, weight- and sex-matched controls [4]. Care is indeed required in selecting regions of interest in which to quantify triacylglycerol in the parenchyma of the pancreas and this underscores the value of using an imagingbased method.

Third, the authors state that they wanted to assess fat distribution in the pancreas in three supposedly different regions [1]. To do this they employed two very different methods, modified Dixon imaging and spectroscopy. This introduces differences related to the methods. Yet the assessment would more simply be carried out by use of a properly established three-point Dixon technique. Using this method, the region of 

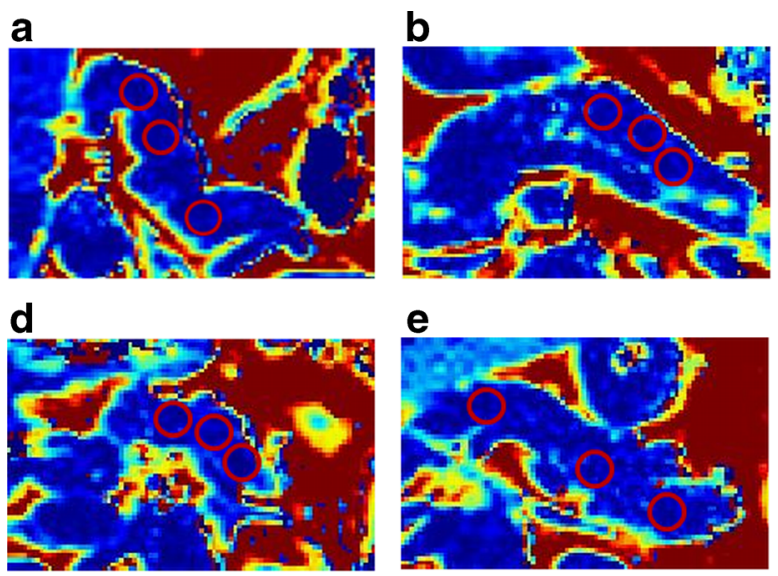

e

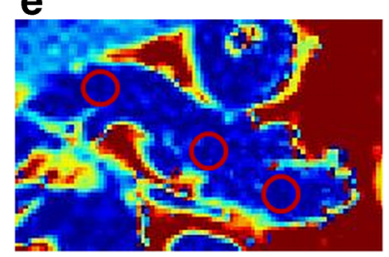

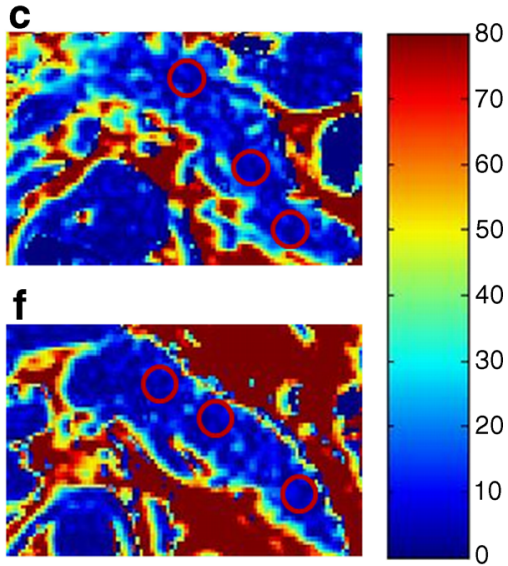

Fig. 1 Fat distribution in the pancreases of people with type 2 diabetes. Colour map of fat distribution in pancreatic slices constructed from Dixon scan of six individuals with type 2 diabetes $(\mathbf{a}-\mathbf{f})$. The colour key is shown on the right. Black represents $0 \%$ fat and colour change represents increasing triacylglycerol concentration. Substantial areas of adipose tissue, such as visceral adipose tissue, are shown as dark red. Also shown are the $3 \times 100 \mathrm{~mm}^{2}$ regions of interest within which the percentage triacylglycerol content is derived (red circles). The average of three regions of interest within each pancreas is taken to derive the percentage pancreas triacylglycerol for each individual. This ranged from $3.3 \%$ to $6.2 \%$ interest can be defined after acquisition to include the whole pancreas, one large area within the pancreatic substance or one or more very small areas. This would have allowed a direct comparison between the three types of regions of interest defined in Fig. 2 of Begovatz et al, using one methodology without raising the issue of the accidental inclusion of visceral fat in the spectroscopic measurements. We have employed this technique not only in pancreas but also, for example, in skeletal muscle in muscular dystrophy $[5,6]$.

The technical problems with the MR methods employed by Begovatz et al underlie their conclusion that the pancreas parenchyma is void of fat. However, this conclusion is comprehensively dismissed by the careful histological studies of human pancreas which have avoided post-mortem artefacts. The pancreases studied by Pinnick et al [7] were retrieved for potential transplantation, hence ex vivo viability was maintained. They observed that there were intracellular fat droplets widely distributed within the exocrine pancreatic cells, and that isolated adipocytes were present and also widely scattered. This mirrors the observations in pancreases of experimental animals [7]. In rodent models, it has been clearly established that triacylglycerol accumulation occurs in synchrony within islets and within the exocrine pancreatic cells during the onset of diet-induced type 2 diabetes [8]. Separately, it is known that human islets avidly take up fatty acids and accumulate triacylglycerol when exposed to a modest increase $(0.33 \mathrm{mmol} / \mathrm{l})$ in extracellular fatty acid concentration [9]. This is also seen in vivo during high-fat feeding in rodents [10].

The three serious errors in MR methodology together with histological and experimental observations indicate that the conclusions of Bergovitz and colleagues are flawed. Triacylglycerol is clearly distributed throughout the parenchyma of the pancreas at a low level and local lipolysis is likely to bring about interstitial and intracellular concentrations of fatty acids sufficient to inhibit beta cell function. The further study of this in relation to the onset and reversal of type 2 diabetes may be of central importance to understanding the condition.

Funding The work was funded by the National Institute for Health Research Newcastle Biomedical Research Centre, The Newcastle Healthcare Charity and a NovoNordisk Foundation UK Research Clinical Fellowship (SS).

Duality of interest The authors declare that there is no duality of interest associated with this manuscript.

Contribution statement KGH developed the methodology, analysed data and co-wrote the manuscript. AAM analysed data, assembled the figure and edited the manuscript. SS analysed data, contributed to discussion and edited the manuscript. RT conceived the study, evaluated data, co-wrote the manuscript and is the guarantor of the work. All approved the final version.

\section{References}

1. Begovatz P, Koliaki C, Weber K et al (2015) Pancreatic adipose tissue infiltration, parenchymal steatosis and beta cell function in humans. Diabetologia 58:1646-1655

2. Lim EL, Hollingsworth KG, Aribisala BS, Chen MJ, Mathers JC, Taylor R (2011) Reversal of type 2 diabetes: normalisation of beta cell function in association with decreased pancreas and liver triacylglycerol. Diabetologia 54:2506-2514

3. Hu HH, Kim HW, Nayak KS, Goran MI (2010) Comparison of fatwater MRI and single-voxel MRS in the assessment of hepatic and pancreatic fat fractions in humans. Obesity 18:841-847

4. Macauley M, Percival K, Thelwall PE, Hollingsworth KG, Taylor R (2015) Altered volume, morphology and composition of the pancreas in type 2 diabetes. PLoS One 10:e0126825 
5. Hollingsworth KG (2014) Quantitative MRI in muscular dystrophy: an indispensable trial endpoint? Neurology 83:956-957

6. Hollingsworth KG, Higgins DM, McCallum M, Ward L, Coombs A, Straub V (2014) Investigating the quantitative fidelity of prospectively undersampled chemical shift imaging in muscular dystrophy with compressed sensing and parallel imaging reconstruction. Magn Reson Med 72:1610-1619

7. Pinnick KE, Collins SC, Londos C, Gauguier D, Clark A, Fielding BA (2008) Pancreatic ectopic fat is characterized by adipocyte infiltration and altered lipid composition. Obesity $16: 522-530$
8. Lee Y, Lingvay I, Szczepaniak LS, Ravazzola M, Orci L, Unger RH (2010) Pancreatic steatosis: harbinger of type 2 diabetes in obese rodents. Int J Obes (Lond) 34:396-400

9. Lalloyer F, Vandewalle B, Percevault F et al (2006) Peroxisome proliferator-activated receptor alpha improves pancreatic adaptation to insulin resistance in obese mice and reduces lipotoxicity in human islets. Diabetes 55:1605-1613

10. Dobbins RL, Szczepaniak LS, Myhill J et al (2002) The composition of dietary fat directly influences glucose-stimulated insulin secretion in rats. Diabetes 51:1825-1833 